\title{
ANKRD26 Gene Mutation
}

National Cancer Institute

\section{Source}

National Cancer Institute. ANKRD26 Gene Mutation. NCI Thesaurus. Code C151909.

A change in the nucleotide sequence of the ANKRD26 gene. 